\title{
Pelagra: Correlação entre diagnóstico e o quadro clínico mais prevalente no Brasil
}

\author{
Pellagra: Correlation between diagnosis and the most prevalent clinical presentation in Brazil \\ Pelagra: Correlación entre diagnóstico y cuadro clínico más prevalente en Brasil
}

Recebido: 01/07/2021 | Revisado: 06/07/2021 | Aceito: 09/07/2021 | Publicado: 21/07/2021

\author{
Rodrigo Sevilla Noleto \\ ORCID: https://orcid.org/0000-0002-7736-9848 \\ Universidade CEUMA, Brasil \\ E-mail: rodrigo.noleto@me.com \\ Nathalia Silva Pinto \\ ORCID: https://orcid.org/0000-0001-6356-1583 \\ Universidade CEUMA, Brasil \\ E-mail: nahsilvap@hotmail.com \\ Janine Silva Ribeiro Godoy \\ ORCID: https://orcid.org/0000-0001-5587-0896 \\ Universidade CEUMA, Brasil \\ E-mail: janinesrg@gmail.com
}

\begin{abstract}
Resumo
Objetivo: Identificar se há dificuldade no diagnóstico da patologia em razão das variadas manifestações clínicas e definir se há uma causa mais prevalente. Método: O estudo consiste em uma Revisão Integrativa de literatura, em que foram selecionados artigos das bases de dados: SciELO e MEDLINE, utilizando como critérios de inclusão artigos em português, inglês e francês, publicados entre 1922-2012; que abordassem o viés etiopatogênico da pelagra; e com qualquer delineamento metodológico. Foram utilizados os descritores: "pelagra", "niacina" e "manifestações clínicas". A amostra final foi constituída de 6 artigos. Resultados: Observou-se nos artigos que compõem a amostra que uma porcentagem alta de pacientes apresentam a "Pelagra Sine Pelagra", ou seja, sem a manifestação das lesões dermatológicas características da pelagra, observando-se desse modo somente os distúrbios gastrointestinais e/ou neurológicos, dificultando o diagnóstico da patologia. No tocante à causa prevalente, é possível definir o alcoolismo como o fator de risco principal para o surgimento da doença. Conclusão: Concluiu-se que o perfil mais relatado foi o de indivíduos oligossintomáticos, em especial pacientes que apresentam quadros de "Pelagra Sine Pelagra". A doença inicia-se com desordens dos órgãos digestivos, seguido do surgimento de lesões cutâneas, e, por fim, alterações nervosas. Em decorrência desse quadro arrastado e de nem sempre ser possível identificar a tríade clássica nos pacientes, tem-se um empecilho no diagnóstico tanto precoce quanto a longo prazo. Ademais, identificou-se alguns fatores de risco para a patologia, como alcoolismo, fator sazonal, radiação solar e desnutrição, sendo o alcoolismo a maior causa de pelagra na atualidade.
\end{abstract}

Palavras-chave: Pelagra; Niacina; Manifestações clínicas.

\begin{abstract}
Objective: To identify if there is difficulty in diagnosing the pathology due to the varied clinical manifestations and to define if there is a more prevalent cause. Method: The study is an Integrative Literature Review, in which articles were selected from the databases: SciELO and MEDLINE, using as inclusion criteria articles in Portuguese, English and French, published between 1922-2012; addressing the etiopathogenic bias of pellagra; and with methodological delimitation. The descriptors used were: "pellagra", "niacin" and "clinical manifestations". The final sample consisted of 6 articles. Results: It was observed that a high percentage of patients present the "Pelagra Sine Pelagra", that is, without the manifestation of the dermatological lesions characteristic of the pellagra, thus observing only the gastrointestinal and/or neurological disorders, making diagnosis of the pathology difficult. About the prevalent cause, alcoholism is the main risk factor for the onset of the disease. Conclusion: It is understood that the most reported profile was that of oligosymptomatic individuals, especially patients who present "Pelagra Sine Pelagra". The disease begins with disorders of digestive organs, followed by the appearance of skin lesions, and, finally, nerve alterations. As a result of this sluggish presentation and the fact that it is not always possible to identify the classic triad in patients, there is an obstacle in the diagnosis, both early and in the long term. In addition, some risk factors for pathology have been identified, such as alcoholism, seasonal factors, solar radiation and malnutrition, with alcoholism being the main cause of pellagra nowadays.
\end{abstract}

Keywords: Pellagra; Niacin; Clinical manifestations. 


\begin{abstract}
Resumen
Objetivo: Identificar si existe dificultad en el diagnóstico de la patología debido a las diversas manifestaciones clínicas y definir si existe una causa más prevalente. Método: El estudio consiste en una revisión integradora de la literatura, en la que se seleccionaron artículos de las bases de datos: SciELO y MEDLINE, utilizando como criterios de inclusión artículos en portugués, inglés y francés, publicados entre 1922-2012; que abordó el sesgo etiopatogénico de la pelagra; y con cualquier diseño metodológico. Se utilizaron los siguientes descriptores: "pelagra", "niacina" y "manifestaciones clínicas". La muestra final estuvo formada por 6 artículos. Resultados: Se observó que un alto porcentaje de pacientes presentan "Pelagra Sine Pelagra", o sea, sin la manifestación de las lesiones dermatológicas características de la pelagra, observándose así solo trastornos gastrointestinales y/o neurológicos, haciéndolo difícil el diagnóstico. Sobre la causa prevalente, el alcoholismo es el principal factor de riesgo para la aparición de la enfermedad. Conclusión: Se concluyó que el perfil más reportado fue el de individuos oligosintomáticos, especialmente pacientes con condiciones de "Pelagra Sine Pelagra". La enfermedad comienza con trastornos de los órganos digestivos, seguidos de la aparición de lesiones cutáneas, y finalmente alteraciones nerviosas. Como resultado de esta condición de que no siempre es posible identificar la tríada clásica en los pacientes, existe un obstáculo para el diagnóstico. Además, se identificaron algunos factores de riesgo para la enfermedad, como el alcoholismo, los factores estacionales, la radiación solar y la desnutrición, siendo el alcoholismo la principal causa de la pelagra en la actualidad.
\end{abstract}

Palabras clave: Pelagra; Niacina; Manifestaciones clínicas.

\title{
1. Introdução
}

A pelagra, uma doença caracterizada pela deficiência de Niacina (B3), vitamina hidrossolúvel que atua nas reações metabólicas de oxirredução e na produção de energia, também atua como componente do dinucleótido de nicotinamida-adenina (NAD+) (Palhares, et al., 2012). De acordo com Krehl WA (1981), a falta de triptofano (aminoácido essencial que pode ser transformado em niacina através dos tecidos), assim como, o excesso de leucina, são fatores que podem inibir a síntese de niacina e consequentemente acarretar no quadro de pelagra.

Segundo Goldberg e Tanner (1922), essa patologia foi descrita pela primeira vez em 1762 por Gaspar Casal, médico particular do rei Felipe V da Espanha, o qual definiu como resultante da ingestão de milho deteriorado, levando vários investigadores a procurar agentes infecciosos e tóxicos neste alimento. Mais de cem anos depois, em 1907 foi descrita pela primeira vez nos Estados Unidos, por Searcy. No entanto, somente em 1914, realizou-se um estudo epidemiológico em doentes mentais, concluindo-se que a pelagra decorria da deficiência de um fator nutricional, denominado de fator P-P (Prevenção da Pelagra). Enfim, em meados do ano de 1937 identificou-se que o consumo de ácido nicotínico fazia regredir significamente a doença (Fouts, et al., 1937).

Atualmente, essa patologia tem sido considerada um déficit de nicotinamida no interior da célula, seja por causa primária (déficit nutricional) ou secundária (doença subjacente) (Júnior, et al., 2008). Esse composto é um importante componente das coenzimas NAD (nicotinamida adenina dinucleotídeo) e NADP (nicotinamida adenina dinucleotídeo fosfato), que funcionam como transportadoras de hidrogênio, sendo elementos fundamentais no processo de oxi-redução celular e exercem papel fundamental no metabolismo dos compostos orgânicos, tais como carboidratos, proteínas e lipídeos, além de atuarem no processo de síntese dos hormônios adrenocorticais a partir da acetilcoenzima-A na desidrogenação do álcool etílico e na conversão do ácido lático em ácido pirúvico. Participando também no ciclo do ácido cítrico, processo este responsável pelo fornecimento de energia à célula. (Júnior, et al., 2008).

De acordo com Hendryx (1991), alterações no processo de respiração celular afetam inicialmente órgãos de alta demanda metabólica como o cérebro e o turnover celular (pele e mucosas), justificando o porquê de tais órgãos serem os principais acometidos pela doença.

Devido ao organismo ser capaz de transformar o aminoácido triptofano em niacina, uma dieta pobre desse aminoácido pode ser uma das causas do desenvolvimento da pelagra. Uma dieta composta por milho, vegetal que é desprovido de tal componente, o alcoolismo, situação onde geralmente a dieta está comprometida, além de, quando prolongado, interferir no metabolismo e absorção da niacina, também podem representar situações que propiciam o desenvolvimento da patologia (Júnior, 
et al., 2008). Além disso, determinados fármacos, tais como a hidrazida ácido nicotínico, podem interferir no metabolismo da niacina e, com isso, acabam por causar sua deficiência no organismo (Russel, 1993).

Quando se instalada através de uma patologia primária, tem-se como agente causador a síndrome do carcinóide (doença de Hartnup), em que o triptofano é utilizado para a síntese de serotonina, podendo levar a um quadro semelhante ao da pelagra (Schumtz, et al., 1987).

A pelagra, caracterizada pela tríade clássica de dermatite, diarreia e demência, se não forem tratados, os pacientes evoluem para óbito. Alguns autores, adicionam "morte" (death em inglês) à tríade, formando assim "4 D's" (Manzella, 2008). Manifesta-se por evolução crônica, com períodos de exacerbação e remissão.

De hábito, a doença inicia com desordens dos órgãos digestivos, em seguida surgem, e às vezes até mesmo anteriormente, as lesões cutâneas, que começam por eritema nas partes expostas à luz e, mais tarde, alterações de maior profundidade na pele. Por fim, só de modo tardio, surgem as alterações nervosas que se evidenciam por fenômenos neuróticos e psicóticos (Marques, 1944).

O quadro cutâneo consiste em eritema nas superfícies expostas ao sol, simétrico, como nas extremidades, face (eritema em asa de borboleta) e ao redor do pescoço ("colar de casal"). Destacando-se como quadros de dermatities a erupção por fotossensibilidade, lesões perineais e espessamento e hiperpigmentação da pele sobre as proeminências ósseas. (Odom, et al., 2000). Acredita-se que a fototoxicidade seja causada pela formilcinurenina e o ácido quinurênico, produtos de degradação do triptofano na via da quinurenina (Walrant, et al., 1975). Quanto aos distúrbios gastrintestinais mais importantes são diarréia e vômitos. Já as alterações neuropsiquiátricas variam desde sintomas leves como astenia, dores variadas, insônia, depressão até total instabilidade emocional e manias (Schumtz, et al., 1987).

Apesar de grandes estudos em relação a patologia, até onde se sabe, pouco se tem descrito correlacionando diagnóstico e quadro clínico. Nesse interim, o objetivo desse trabalho foi identificar se há dificuldade no diagnóstico da patologia em razão das variadas manifestações clínicas e definir se há uma causa mais prevalente.

\section{Metodologia}

O presente estudo consiste em uma revisão integrativa, sendo esta um método de pesquisa importante por possuir a finalidade de reunir e sintetizar resultados de pesquisas sobre um delimitado tema ou questão, de maneira sistemática e ordenada, contribuindo para o aprofundamento do conhecimento do tema investigado. É relatada como método de pesquisa na literatura desde 1980 (Roman \& Friedlander, 1998).

Para a elaboração da mesma, as seguintes etapas foram percorridas: estabelecimento da hipótese e objetivos da revisão integrativa; estabelecimento de critérios de inclusão e exclusão de artigos (seleção da amostra); definição das informações a serem extraídas dos artigos selecionados; análise dos resultados; discussão e apresentação dos resultados e a última etapa consistiu na apresentação da revisão.

Para guiar esta revisão integrativa, formulou-se as seguintes questões: há dificuldade no diagnóstico precoce da patologia em decorrência da variedade de manifestações? Entre as causas primárias e secundárias, há uma que apresente maior prevalência? Há uma faixa etária mais afetada?

Para a seleção dos artigos foram utilizadas duas bases de dados, a saber: SciELO (Scientific Electronic Library Online) e o MEDLINE (Medical Literature Analysis and Retrieval Sistem on-line). Dessa forma, procurou-se ampliar o âmbito da pesquisa, minimizando possíveis vieses nessa etapa do processo de elaboração da revisão integrativa.

Os critérios de inclusão dos artigos definidos, inicialmente, para a presente revisão integrativa foram: artigos publicados em português, inglês e francês com os resumos disponíveis nas bases de dados selecionadas, no período compreendido entre 1922-2012; artigos que abordassem o viés etiopatogênico da pelagra; e artigos com qualquer delineamento metodológico. Foram 
utilizados os seguintes descritores: "pelagra", "niacina" e "manifestações clínicas". A busca foi realizada pelo acesso on-line e, utilizando os três critérios de inclusão, a amostra final desta revisão integrativa foi constituída de 6 artigos.

Para a análise e posterior síntese dos artigos que atenderam aos critérios de inclusão foi utilizado um quadro sinóptico especialmente construído para esse fim, que contemplou os seguintes aspectos, considerados pertinentes: nome da pesquisa; nome dos autores; intervenção estudada; resultados; recomendações/conclusões.

\section{Resultados e Discussão}

$\mathrm{Na}$ presente revisão integrativa, analisou-se seis artigos que atenderam aos critérios de inclusão previamente estabelecidos e, a seguir, apresentar-se-á um panorama geral dos artigos avaliados.

Os artigos em estudo foram publicados principalmente nas revistas: Revista da Sociedade Brasileira de Clínica Médica, Revista Médica de Minas Gerais, Revista Científica da Faminas, Revista HCPA e Revista Cogitare Enfermagem. Com relação ao tipo de revista científica na qual foram publicados os artigos selecionados, percebeu-se que todos estavam enquadrados em periódicos da área da saúde, apresentando um conhecimento mais especializado na área dermatológica e neurológica, fator favorável ao estudo, haja vista que reconhecer o quadro clínico e as diferentes manifestações, bem como os fatores de risco mais predisponentes pelo olhar de especialistas auxilia no diagnóstico precoce e condução dos casos clínicos.

Dentre os artigos incluídos na revisão integrativa, dois são de autoria de médicos, um por residentes de dermatologia e médicos, outro redigido por médicos e enfermeiros, um por médicos e biomédicos e um possui entre seus autores somente farmacêuticos.

Em se tratando do tipo de artigo, pode-se observar que quatro eram artigos originais, onde o viés mais utilizado foi o relato de caso; um era proveniente de uma revisão bibliográfica e apenas um não foi possível identificar a origem. Notou-se a falta de artigos oriundos de dissertação e tese de doutorado, o que infelizmente denota uma baixa difusão do tema abordado, uma vez que o mestrado fornece capacitação para a área de pesquisa, fato esse que contribui para a construção, publicação e atualização do conhecimento.

Com relação aos delineamentos de pesquisa mais frequentes nas publicações estudadas, identificou-se que 3 utilizaram a abordagem metodológica qualitativa, 1 desenvolveu estudo com método quantitativo e 2 foram definidos como não pesquisa, incluindo pesquisa bibliográfica e revisão de literatura.

Os descritores mais utilizados pelos autores foram pelagra, presente em todos os artigos, seguido de triptofano, e niacina. Cabe ressaltar que um artigo poderia conter mais de um descritor.

Nas Tabelas 1, 2, e 3 apresenta-se a síntese dos artigos incluídos na presente revisão integrativa. 
Tabela 1: Apresentação da síntese de artigos incluídos na revisão integrativa.

\begin{tabular}{|c|c|c|c|c|}
\hline Ano & Autoria & Objetivo & Resultado & Conclusão \\
\hline 2011 & $\begin{array}{l}\text { Mata, W.S } \\
\text { Silva, I.M } \\
\text { Pereira, I.E } \\
\text { Coelho, L.P } \\
\text { Paiva, E.O.C } \\
\text { Barbosa, V.A }\end{array}$ & $\begin{array}{l}\text { Estudar o caso de uma } \\
\text { paciente de } 35 \text { anos, } \\
\text { procedente de Caratinga } \\
\text { (MG), que se apresentou } \\
\text { ao pronto atendimento } \\
\text { com descamação de pele e } \\
\text { relato de diarréia } \\
\text { freqüente. }\end{array}$ & $\begin{array}{l}\text { Foi dado o diagnóstico de } \\
\text { pelagra e instituído } \\
\text { tratamento que resultou em } \\
\text { melhora do estado físico da } \\
\text { paciente. }\end{array}$ & $\begin{array}{l}\text { Concluiu-se que os sinais } \\
\text { e sintomas clínicos foram } \\
\text { suficientes para o } \\
\text { diagnóstico de pelagra. }\end{array}$ \\
\hline
\end{tabular}

Fonte: Autores.

Tabela 2: Apresentação da síntese de artigos incluídos na revisão integrativa.

\begin{tabular}{|c|c|c|c|c|}
\hline Ano & Autoria & Objetivo & Resultado & Conclusão \\
\hline 2006 & $\begin{array}{l}\text { Salvador, S. } \\
\text { Bruning, G.E } \\
\text { Leitão, C.B }\end{array}$ & $\begin{array}{l}\text { Analisar o caso de um } \\
\text { paciente de } 48 \text { anos, } \\
\text { sexo masculino, com } \\
\text { história de alcoolismo } \\
\text { crônico, diarréia, } \\
\text { insônia, irritabilidade e } \\
\text { diminuição da memória. }\end{array}$ & $\begin{array}{l}\text { No nosso meio, como é o } \\
\text { caso do paciente, o } \\
\text { aparecimento da pelagra } \\
\text { está ligado à desnutrição } \\
\text { decorrente do alcoolismo } \\
\text { crônico. }\end{array}$ & $\begin{array}{l}\text { Um alto grau de } \\
\text { suspeição e o } \\
\text { conhecimento das } \\
\text { características das lesões } \\
\text { típicas da pelagra são } \\
\text { essenciais para o } \\
\text { diagnóstico e tratamento } \\
\text { adequados nas fases } \\
\text { iniciais da doença. }\end{array}$ \\
\hline 1944 & Marques, A. & $\begin{array}{l}\text { São estudadas as várias } \\
\text { questões gerais da } \\
\text { Pelagra, nomeadamente } \\
\text { os aspectos clínicos e } \\
\text { etiopatogênicos. }\end{array}$ & $\begin{array}{l}\text { Concluiu-se que os } \\
\text { aspectos neurológicos da } \\
\text { Pelagra não se encontram } \\
\text { mais vezes, porque, via de } \\
\text { regra, só se diagnostica a } \\
\text { doença quando as lesões } \\
\text { cutâneas são evidentes; de } \\
\text { hábito deixam-se de lado } \\
\text { os exemplares de Pelagra } \\
\text { Sine Pelagra" }\end{array}$ & $\begin{array}{l}\text { Acredita-se ser de grande } \\
\text { importância salientar os } \\
\text { exemplares em que não } \\
\text { se encontram sintomas } \\
\text { cutâneos e configuram a } \\
\text { "Pelagra sine Pelagra". }\end{array}$ \\
\hline 2008 & $\begin{array}{l}\text { Júnior, J.V.O. } \\
\text { Zaccariotto, L.M. } \\
\text { Maciel. J.N. } \\
\text { Sittart, J.A. }\end{array}$ & $\begin{array}{l}\text { Examinar a história, } \\
\text { etiopatogenia, } \\
\text { diagnóstico e tratamento } \\
\text { da Pelagra. }\end{array}$ & $\begin{array}{l}\text { Apesar de apresentar-se } \\
\text { sob a forma de demência, } \\
\text { diarréia e dermatite - três } \\
\text { "Ds", a forma de } \\
\text { apresentação mais comum } \\
\text { é com sintomas } \\
\text { incompletos e não a tríade } \\
\text { completa. }\end{array}$ & $\begin{array}{l}\text { A pelagra não é uma } \\
\text { doença tão comum no } \\
\text { nosso meio. Atenção } \\
\text { diagnóstica deve ser } \\
\text { dada para as formas } \\
\text { incompletas que se } \\
\text { manifestam unicamente } \\
\text { por sintomas } \\
\text { neurológicos, } \\
\text { cutâneos ou ainda } \\
\text { gastrintestinais. }\end{array}$ \\
\hline
\end{tabular}

Fonte: Autores. 
Tabela 3: Apresentação da síntese de artigos incluídos na revisão integrativa.

\begin{tabular}{|c|c|c|c|c|}
\hline Ano & Autoria & Objetivo & Resultado & Conclusão \\
\hline 2012 & $\begin{array}{l}\text { Palhares, D.M.F. } \\
\text { Albuquerque, N.N. } \\
\text { Foureaux, F.S. } \\
\text { Pereira, F.S. } \\
\text { Alkmin, M.B.M. } \\
\text { Leite, J.A. }\end{array}$ & $\begin{array}{l}\text { Identificar o } \\
\text { diagnóstico mais } \\
\text { provável com base na } \\
\text { história clínica e na } \\
\text { análise das imagens } \\
\text { apresentadas. }\end{array}$ & $\begin{array}{l}\text { A presença de lesões } \\
\text { hipercrômicas, } \\
\text { descamativas, } \\
\text { pruriginosas e bem } \\
\text { delimitadas em áreas de } \\
\text { exposição solar, associada } \\
\text { com história social de } \\
\text { alcoolismo, sugere } \\
\text { Pelagra como diagnóstico } \\
\text { mais provável. }\end{array}$ & $\begin{array}{l}\text { O diagnóstico é } \\
\text { clínico, sobretudo na } \\
\text { presença da tríade } \\
\text { clássica, dermatite, } \\
\text { diarreia e demência } \\
\text { (“3 Ds”), sendo a } \\
\text { propedêutica } \\
\text { complementar de } \\
\text { pouca importância. }\end{array}$ \\
\hline 2011 & $\begin{array}{l}\text { Şenormanc1, O. } \\
\text { Oya Güçlü, R.K } \\
\text { Şenormanc1, G. }\end{array}$ & $\begin{array}{l}\text { Analisar a relação } \\
\text { entre Pelagra e o } \\
\text { álcool a partir de } \\
\text { necropsias de } \\
\text { pacientes etilistas } \\
\text { crônicos. }\end{array}$ & $\begin{array}{l}\text { Em } 20 \text { casos foi } \\
\text { identificado achados } \\
\text { clínicos e patológicos } \\
\text { compatíveis com Pelagra. } \\
\text { Nenhum exibiu as } \\
\text { características cutâneas } \\
\text { clássicas da pelagra ou } \\
\text { tinha o diagnóstico } \\
\text { confirmado por métodos } \\
\text { bioquímicos. }\end{array}$ & $\begin{array}{l}\text { Acredita-se que esses } \\
20 \text { casos tiveram } \\
\text { "pelagra" em } \\
\text { fundamentos } \\
\text { neuropatológicos e } \\
\text { clínicos. }\end{array}$ \\
\hline
\end{tabular}

Fonte: Autores.

Com relação à coerência dos resultados com os objetivos, todos os artigos responderam aos seus. O objetivo é fundamental para a compreensão do artigo publicado, além de facilitar a leitura do mesmo, devendo estar em concordância com as variáveis investigadas.

Dos seis artigos selecionados, três abordaram o perfil subclínico (“Pelagra Sine Pelagra”) em seu conteúdo. Dentre eles, dois identificaram este perfil como sendo o de maior prevalência na população estudada e o outro alertou para a baixa detecção do paciente oligossintomático, fator preocupante no que tange ao diagnóstico e tratamento da doença. Os outros temas abordados nos demais artigos referiam-se aos fatores que influenciam para a ocorrência de casos de pelagra, como o alcoolismo; fator sazonal, uma vez que há uma maior incidência nos períodos de verão e primavera; radiação solar e desnutrição; bem como a necessidade da capacitação de profissionais de saúde para o reconhecimento precoce e tratamento adequado da doença e suas complicações.

Em relação aos objetivos desta revisão, ou seja, identificar se há dificuldade no diagnóstico da patologia em detrimento das variadas manifestações e definir se há uma causa mais prevalente, observou-se nos artigos que compõem a amostra que uma porcentagem alta de pacientes apresentam a "Pelagra Sine Pelagra", ou seja, as lesões hipercrômicas, descamativas, pruriginosas e bem delimitadas em áreas de exposição solar não se apresentam como manifestações clínicas, observando-se desse modo somente os distúrbios gastrointestinais e/ou neurológicos. Tal fato dificulta o diagnóstico da patologia.

No tocante à causa prevalente, é possível definir o alcoolismo como o fator de risco principal para o surgimento da doença, haja vista que a deficiência de niacina decorrente da desnutrição não é mais uma realidade, e que a taxa de etilismo crônico da sociedade é alta. Em relação à faixa etária mais prevalente, observou-se que nos relatos de caso analisados os pacientes possuíam idades entre 35 e 54 anos, fato que nos leva a indagar a respeito de sua relação com a cronicidade do alcoolismo.

Conforme os resultados expostos, percebeu-se a ausência de publicação em periódicos específicos sobre o tema abordado na área dermatológica da medicina. Tal fato pode estar dificultando a rápida atualização desse tipo de conhecimento científico e, consequentemente, a sua divulgação. 
A análise dos resultados das pesquisas evidenciou ainda que urge uma série de medidas necessárias para a capacitação de profissionais de saúde para que haja o reconhecimento precoce e tratamento adequado da doença e suas complicações.

\section{Conclusão}

Concluindo a presente revisão integrativa, na busca da melhor evidência disponível, referente à correlação entre diagnóstico e o quadro clínico mais prevalente da pelagra no Brasil, entende-se que o perfil mais relatado foi o de indivíduos oligossintomáticos, em especial pacientes que apresentam o quadros de "Pelagra Sine Pelagra". De hábito, a doença inicia-se com desordens dos órgãos digestivos (glossite, alterações do faringe, fenômenos gastrintestinais - especialmente diarréia); seguido do surgimento de lesões cutâneas, e, por fim, normalmente, só de modo tardio, alterações nervosas que se evidenciam por fenômenos neuróticos e psicóticos.

Em decorrência desse quadro arrastado e de nem sempre ser possível identificar os 3 D's nos pacientes, tem-se um empecilho no diagnóstico, tanto precoce quanto a longo prazo, da patologia. Ademais, identificou-se alguns fatores de risco para a patologia, tais como alcoolismo, fator sazonal, radiação solar e desnutrição; destes, todos os artigos alertaram para o alcoolismo como a maior causa de pelagra na atualidade.

Avaliando ainda a proposta inicial desta revisão, observa-se dificuldades na investigação relacionadas ao território abordado. Apesar da palavra-chave selecionada incluir todas as regiões do país, somente se localizou artigos pontuais, referentes a poucos casos, e em períodos diferentes. Desse modo, há dificuldade em visualizar o panorama atual do país com precisão.

Frente às lacunas evidenciadas e aos resultados apontados nos artigos incluídos nesta revisão integrativa, entende-se ser necessário intensificar esforços para o desenvolvimento de pesquisas com delineamentos que produzam evidências fortes relativas ao tema investigado, haja vista que a pelagra é uma doença grave e apresenta letalidade ainda elevada em todas as faixas etárias se não houver correto diagnóstico.

Trabalhos que abordem esse tema se tornam relevantes pelo caráter preventivo que podem possuir, ajudando a identificar diagnósticos mais precoces, possibilitando o início do tratamento antes de sequelas graves, e por muitas vezes irreversíveis.

\section{Referências}

Filgueiras, F. M., Stolarczuk, D. A., Gripp, A. C., \& Succi, I. C. (2011). Benign symmetrical lipomatosis and pellagra associated with alcoholism. An Bras Dermatol, 86(6):1189-92.

Fouts, P., Helmer, O. M., Lepkovsky, S., \& Jukesth (1937). Treatment of human pellagra with nicotinic acid. Proc.Soc. Exp. Blot. Med., 37:405-7.

Frankenburg, F. R. (2009). Vitamin discoverieds and disasters: History, science \& controversies. Praeger/ABC-CLIO.

Goldberger, J., \& Tanner, W. F. (1922). Amino acid deficiency probably the primary etiological factor in pellagra. Pub Health Rep., 37:462-86.

Hegyi J., Schwartz, R. A., \& Hegyi, V. (2004). Pellagra: Dermatitis, dementia, and diarrhea. Int J Dermatol, 43:1-5.

Hendryx, W. M. (1991). Pellagra and pellagra like dermatoses. Etiology, differential diagnosis, dermatopathology and treatment. Semin Kermatol, 10:282- 92.

Júnior, J. V. O., Zaccariotto, L. M., Maciel, J. N., \& Sittart, J. A. (2008). Pelagra. Rev Soc Bra Clin Med., 6(4): 139-141

Krehl, W. A. (1981). Discovery of the effect of tryptofan on niacin deficiency. Fed Proc., 40(5):1527-30.

Manzella, F. (2008). Pellagra: A biocultural perspective. P 1-12.

Marques, A. (1944). Aspectos neurológicos da pelagra. Conferência feita na Sociedade de Medicina e Cirurgia de S. Paulo.

Mata, W. S., et al. (2011). Relato de caso: pelagra. Revista Científica da Faminas - V. 7, N. 2.

Odom, R. B., James, W. D., Berger, T. G (2000). Andrews' Disease of the Skin. (9.ed.) 610-11, WB Saunders Company.

Palhares, D. M. F., Albuquerque, N. N., Foureaux, F. S., Pereira, F. S., Alkmin, M. B. M., \& Leite, J. A. Caso 6. Rev Med Minas Gerais, 22(2): 246-248 
Research, Society and Development, v. 10, n. 9, e7210917787, 2021

(CC BY 4.0) | ISSN 2525-3409 | DOI: http://dx.doi.org/10.33448/rsd-v10i9.17787

Roman, A. R., \& Friedlander, M. R (1998). Revisão integrativa de pesquisa aplicada à enfermagem. Cogitare Enferm., 3(2):109-12.

Russel, R. M. (1993). Doenças nutricionais parte 15. In: Tratado de Medicina Interna, (19a ed.), (pp. 1167-1215) Guanabara Koogan: RJ.

Salvador, S., bruning, G. E., \& Leitão, C (2006). Pelagra. Rev HCPA, 26(2)

Schumtz, J. L., et al. (1987). Les èrythemes pellagroids medicamentaux. Une observation d'erytheme pellagroide sencondaire à isoniazida. Ann Dermatol Venereol,114(4):569-76.

Şenormanci, O., Oya güçlü, R. K., \& ŞenormancI, G (2011). A Case of Pellagra Associated with Long Term Alcoholism. Düşünen Adam The Journal of Psychiatry and Neurological Sciences, 24:251-252.

Stratigos J. D., \& Katsambas A (1977). Pellagra: a still existing disease. Br J. Dermatol, 96:99-106.

Walrant, P., Santus, R., \& Grossweiner, L. I. (1975). Photosensitizing properties of N-formylkynurenine. Photochem Photobiol, 22:63-5. 